\title{
Quantum gates and quantum algorithms with Clifford algebra technique
}

\author{
M. Gregorič, N.S. Mankoč Borštnik \\ Department of Physics, University of Ljubljana, Jadranska 19, 1000 Ljubljana,
}

(Dated: December 1, 2018)

\begin{abstract}
We use the Clifford algebra technique [1, 2], that is nilpotents and projectors which are binomials of the Clifford algebra objects $\gamma^{a}$ with the property $\left\{\gamma^{a}, \gamma^{b}\right\}_{+}=2 \eta^{a b}$, for representing quantum gates and quantum algorithms needed in quantum computers in an elegant way. We identify $n$ qubits with spinor representations of the group $S O(1,3)$ for a system of $n$ spinors. Representations are expressed in terms of products of projectors and nilpotents. An algorithm for extracting a particular information out of a general superposition of $2^{n}$ qubit states is presented. It reproduces for a particular choice of the initial state the Grover's algorithm [3]].
\end{abstract}




\section{INTRODUCTION}

It is easy to prove (and it is also well known) that any type of a quantum gate, operating on one qubit and represented by an unitary operator, can be expressed as a product of the two types of quantum gates - the phase gate and the Hadamard's gate - while the C-NOT gate, operating on two quantum bits, enables to make a quantum computer realizable, since all the needed operations can be expressed in terms of these three types of gates. In the references [4, 5] the use of the geometrical algebra to demonstrate these gates and their functioning is presented.

In this paper we use the technique from the references [1, 2], which represents spinor representations of the group $S O(1,3)$ in terms of projectors and nilpotents, which are binomials of the Clifford algebra objects $\gamma^{a}$ and we identify the spinor representation of two one spinor states with the two quantum bits $|0\rangle$ and $|1\rangle$ and accordingly $n$ spinors' representation of $S O(1,3)$ with the $n$-qubits. The three types of the gates can then be expressed in terms of projectors and nilpotents in a transparent and elegant way.

We present also the algorithm for extracting a particular information out of any superposition of a $n$-qubit state. For a particular choice of the initial $n$-qubit state this general algorithm reproduces the Grover's algorithm [3].

\section{THE TECHNIQUE FOR SPINOR REPRESENTATIONS}

We define in this section $\left(2^{4 / 2-1}\right)$ basic states for the spinor (fundamental) representation of the group $S O(1,3)$ by expressing the basic states as polynomials of the Clifford algebra objects $\gamma^{a}$-nilpotents and projectors [1, 2] - chosen to be eigenvectors of the Cartan subalgebra set (with 2 commuting operators) of the 6 infinitesimal operators of the group $S O(1,3)$. We identify one qubit with one of the two spinor basic states, distinguishing between the chiral representation and the representation with a well defined parity. We make at the end use of states with well defined parity, although both representations for spinors are equivalent and the proposed quantum gates work for both representations. We identify $n$-qubits with states which are superposition of $2^{n}$ products of $n$ one spinor states. We also present some relations, useful when defining the quantum gates.

The six infinitesimal generators $S^{a b}$ of the group $S O(1,3)\left(S^{01}, S^{02}, S^{03}, S^{23}, S^{31}, S^{12}\right)$ 
fulfill the Lorentz algebra $\left\{S^{a b}, S^{c d}\right\}_{-}=i\left(\eta^{a d} S^{b c}+\eta^{b c} S^{a d}-\eta^{a c} S^{b d}-\eta^{b d} S^{a c}\right)$. For spinors can the generators $S^{a b}$ be written in terms of the operators $\gamma^{a}$ fulfilling the Clifford algebra

$$
\begin{aligned}
\left\{\gamma^{a}, \gamma^{b}\right\}_{+} & =2 \eta^{a b}, \quad \operatorname{diag}(\eta)=(1,-1,-1,-1) \\
S^{a b} & =\frac{i}{2} \gamma^{a} \gamma^{b}, \quad \text { for } a \neq b \text { and } 0 \text { otherwise }
\end{aligned}
$$

They define the spinor (fundamental) representation of the group $S O(1,3)$. Choosing for the Cartan subalgebra set of commuting operators $S^{03}$ and $S^{12}$, the spinor states

$$
\begin{array}{rlrl}
|0\rangle_{L} & =\left[\begin{array}{cc}
03 & 12 \\
-i
\end{array}\right)(+)\left|\varphi_{0}\right\rangle, & |1\rangle_{L}=(+i)\left[\begin{array}{c}
03 \\
-
\end{array}\right]\left|\varphi_{0}\right\rangle, \\
|0\rangle_{R}=(+i)(+)\left|\varphi_{0}\right\rangle, & |1\rangle_{R}=\left[\begin{array}{ll}
03 & -i 2
\end{array}\right][-]\left|\varphi_{0}\right\rangle,
\end{array}
$$

where $\left|\varphi_{0}\right\rangle$ is a vacuum state[1, 2] (which from now on we shall skip it since its only required property is that when the Clifford algebra objects from Eq.(2) are applied on the vacuum state the left hand side do not give zero) and with nilpotents $(\stackrel{a b}{k} \stackrel{a b}{k}(k)=0)$ and projectors $\begin{array}{cc}a b a b & a b \\ ([k][k]=[k]) & \text { defined as }\end{array}$

$$
\begin{aligned}
& \stackrel{03}{( \pm i}):=\frac{1}{2}\left(\gamma^{0} \mp \gamma^{3}\right), \quad \stackrel{12}{( \pm)}:=\frac{1}{2}\left(\gamma^{1} \pm i \gamma^{2}\right), \\
& {\left[\begin{array}{l}
03 \\
\pm i]
\end{array}:=\frac{1}{2}\left(1 \pm \gamma^{0} \gamma^{3}\right), \quad[ \pm]:=\frac{1}{2}\left(1 \pm i \gamma^{1} \gamma^{2}\right)\right. \text {, }}
\end{aligned}
$$

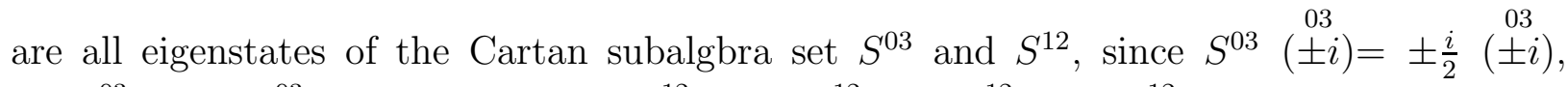
$S^{03}\left[\begin{array}{l}03 \\ \pm i\end{array}\right]= \pm \frac{i}{2}[ \pm i]$ and similarly $S^{12}(\stackrel{12}{ \pm})= \pm \frac{1}{2}(\stackrel{12}{ \pm}), S^{12}[\stackrel{12}{ \pm}]= \pm \frac{1}{2}[ \pm]$, what can very easily be checked just by applying $S^{03}$ and $S^{12}$ on a particular nilpotent $(( \pm i),( \pm))$ or projector $[ \pm i],[ \pm]$ and by using Eq.(11). The states $|0\rangle_{L}$ and $|1\rangle_{L}$ have handedness $\Gamma=-4 i S^{03} S^{12}$ equal to -1 , while the states $|0\rangle_{R}$ and $|1\rangle_{R}$ have handedness equal to 1 , which again can easily be proved just by inspection. We normalize the states as follows [1]

$$
{ }_{\beta}\langle i \mid j\rangle_{\alpha}=\delta_{i j} \delta_{\alpha \beta}
$$

where $i, j$ denote 0 or 1 and $\alpha, \beta$ left and right handedness.

When describing a spinor in its center of mass motion, the representation with a well defined parity is more convenient

$$
\begin{aligned}
& |0\rangle=\frac{1}{\sqrt{2}}\left(\left[\begin{array}{ll}
03 & 12 \\
-i
\end{array}\right](+) \pm\left(\begin{array}{l}
03 \\
+i
\end{array}\right)(+)\right), \\
& |1\rangle=\frac{1}{\sqrt{2}}\left((++i)[-] \pm\left[\begin{array}{l}
03 \\
{[-i][-]}
\end{array}{ }^{12} .\right.\right.
\end{aligned}
$$


Usually spinors with a positive parity are identified with particles, while spinors with a negative parity are identified with antiparticles. For our purpose parity property does not matter. We can use any of these two types of states. Nilpotents and projectors fulfil the following relations [1, 2] (which can be checked just by using the definition of the nilpotents and projectors $(\mathrm{Eq}, \sqrt[3]{3})$ and by taking into account the Clifford property of $\gamma^{a}$ 's (Eq, 1)

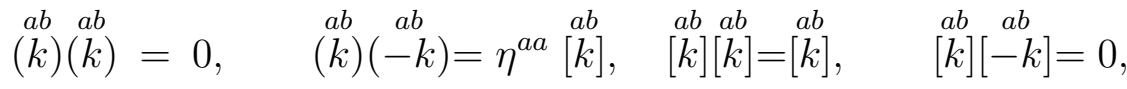

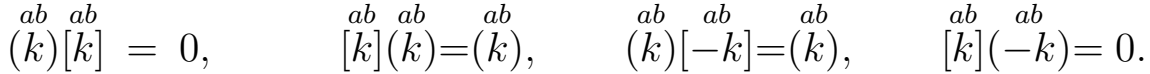

We then find that the operators

$$
\left.\tau_{L}^{\mp}:=-(\stackrel{03}{\mp} \stackrel{12}{\mp} \stackrel{12}{\mp}), \quad \tau_{R}^{\mp}:=(\stackrel{03}{\mp i}) \stackrel{12}{\mp}\right)
$$

transform a state of a particular handedness (left or right) into a state of the same handedness or annihilate it, while they annihilate the states of an opposite handedness

$$
\begin{aligned}
\tau_{L}^{-}|0\rangle_{L} & =|1\rangle_{L}, \quad \tau_{L}^{+}|1\rangle_{L}=|0\rangle_{L}, \\
\tau_{R}^{-}|0\rangle_{R} & =|1\rangle_{R}, \quad \tau_{R}^{+}|1\rangle_{R}=|0\rangle_{R} .
\end{aligned}
$$

All the other applications $\tau_{L}^{\mp}$ and $\tau_{R}^{\mp}$ give zero.

We also find that the operators

$$
\tau^{\mp}:=\tau_{L}^{\mp}+\tau_{R}^{\mp}=-(\stackrel{03}{ \pm} \stackrel{12}{\mp} \stackrel{12}{\mp})+(\stackrel{03}{\mp i} \stackrel{12}{\mp} \stackrel{12}{\mp})
$$

transform a state of a well defined parity (Eq 5 ) into a state of the same parity or annihilate it

$$
\tau^{-}|0\rangle=|1\rangle, \quad \tau^{+}|1\rangle=|0\rangle,
$$

while the rest of applications give zero, accordingly $\left(\tau^{+}+\tau^{-}\right)|0\rangle=|1\rangle,\left(\tau^{+}+\tau^{-}\right)|1\rangle=|0\rangle$.

We present the following useful properties of $\tau^{ \pm}$, valid for $\tau_{L}^{ \pm}$and $\tau_{R}^{ \pm}$as well, so that we shall from now on skip the index $L, R$,

$$
\begin{aligned}
& \left(\tau^{ \pm}\right)^{2}=0, \quad\left(\tau^{ \pm}\right)^{\dagger}=\tau^{\mp}, \quad \tau^{+} \tau^{-}=[+], \quad \tau^{-} \tau^{+}=[-], \quad\left(\tau^{+}+\tau^{-}\right)^{2}=I, \\
& \tau^{+}\left[\begin{array}{l}
12 \\
+
\end{array}\right]=0, \quad \tau^{-}\left[\begin{array}{l}
12 \\
-
\end{array}=0, \quad[+] \tau^{-}=0, \quad[-] \tau^{+}=0,\right. \\
& \tau^{+}[\stackrel{12}{-}]=\tau^{+}, \quad \tau^{-}[\stackrel{12}{+}]=\tau^{-}, \quad[+12] \tau^{+}=\tau^{+}, \quad[\stackrel{12}{-}] \tau^{-}=\tau^{-} .
\end{aligned}
$$


A $n$-qubit state can be written in the chiral representation as

$$
\left|i_{1} i_{2} \cdots i_{l} \cdots i_{n}\right\rangle_{\alpha}=\prod_{l=1, n}\left|i_{l}\right\rangle_{\alpha}, \quad \alpha=L, R
$$

while in the representation with well defined parity we similarly have

$$
\left|i_{1} i_{2} \cdots i_{l} \cdots i_{n}\right\rangle=\prod_{l=1, n}\left|i_{k}\right\rangle
$$

$i_{l}$ stands for $|0\rangle_{l}$ or $|1\rangle_{l}$. All the raising and lowering operators $\tau_{l}^{\alpha \pm}, \alpha=L, R$ or $\tau_{l}^{ \pm}$carry the index of the corresponding qubit manifesting that they only apply on the particular $k$ state, while they do not "see" all the other states. Since they are made out of an even number of the Clifford odd nilpotents, they do not bring any sign when jumping over one-qubit states.

Either in the chiral representation or in the representation with well defined parity basic states are the eigenstates of the operators $S^{12}$. According to Eq.(10) any $n$-qubit state can be written as follows

$$
\left|i_{1} i_{2} \cdots i_{l} \cdots i_{n}\right\rangle_{\alpha}=\prod_{l=1, n}\left(\tau_{l}^{-}\right)^{i_{l}}|0\rangle_{l}
$$

with $i_{l}$ equal to 0 for the state with the eigenvalue of $S^{12}$ equal to $\frac{1}{2}$ or 1 for the state with the eigenvalue of $S^{12}$ equal to $-\frac{1}{2}$.

\section{QUANTUM GATES}

We define in this section three kinds of quantum gates: the phase gate and the Hadamard's gate, which apply on a particular qubit $l$ and the C-NOT gate, which applies on two qubits, say $l$ and $m$. All three gates are expressed in terms of projectors and an even number of nilpotents.

i. The phase gate $\mathcal{R}_{\Phi_{l}}$ is defined as

$$
\mathcal{R}_{\Phi_{l}}=\left[+[]_{l}+e^{i \Phi_{l}}[-12]_{l} .\right.
$$

Statement: The phase gate $\mathcal{R}_{\Phi_{l}}$ if applying on $\left|0_{l}\right\rangle$ leaves it in the same state $\left|0_{l}\right\rangle$ without any change, while if applying on $\left|1_{l}\right\rangle$ multiplies this state with $e^{i \Phi}$. This is true for states with well defined parity $\left|i_{l}\right\rangle$ and also for the states with well defined handedness $\left(\left|i_{l}\right\rangle_{L}\right.$ and $\left.\left|i_{l}\right\rangle_{R}\right)$. 
Proof: To prove this statement one only has to apply the operator $\mathcal{R}_{\Phi_{l}}$ on $\left|i_{l}\right\rangle,\left|i_{l}\right\rangle_{L}$ and $\left|i_{l}\right\rangle_{R}$, with $i_{l}$ equal to 0 or 1 , taking into account equations from Sect. II],

ii. The Hadamard's gate $\mathcal{H}_{l}$ is defined as

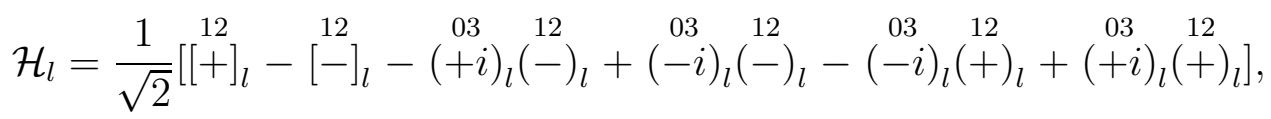

or equivalently in terms of $\tau^{ \pm}$(Eq.(9) )

$$
\mathcal{H}_{l}=\frac{1}{\sqrt{2}}\left[[+]_{l}^{12}-[-]_{l}+\tau_{l}^{-}+\tau_{l}^{+}\right] .
$$

Statement: The Hadamard's gate $\mathcal{H}_{l}$ if applying on $\left|0_{l}\right\rangle$ transforms it to $\left(\frac{1}{\sqrt{2}}\left(\left|0_{l}\right\rangle+\left|1_{l}\right\rangle\right)\right)$, while if applying on $\left|1_{l}\right\rangle$ it transforms the state to $\left(\frac{1}{\sqrt{2}}\left(\left|0_{l}\right\rangle-\left|1_{l}\right\rangle\right)\right)$. This is true for states with well defined parity $\left|i_{l}\right\rangle$ and also for the states in the chiral representation $\left|i_{l}\right\rangle_{L}$ and $\left|i_{l}\right\rangle_{R}$.

Proof: To prove this statement one only has to apply the operator $\mathcal{H}_{l}$ on $\left|i_{l}\right\rangle,\left|i_{l}\right\rangle_{L}$ and $\left|i_{l}\right\rangle_{R}$, with $i_{l}$ equal 0 or 1 , taking into account equations from Sect. II]

iii. The C-NOT gate $\mathcal{C}_{l m}$ is defined as

$$
\left.\mathcal{C}_{l m}=[\stackrel{12}{+}]_{l}+[\stackrel{12}{-}]_{l}\left[-(\stackrel{03}{+} i)_{m}(\stackrel{12}{-})_{m}+\stackrel{03}{(-i}\right)_{m}(\stackrel{12}{-})_{m}-(\stackrel{03}{-i})_{m}(\stackrel{12}{+})_{m}+(\stackrel{03}{+i})_{m}(\stackrel{12}{+})_{m}\right]
$$

or equivalently

$$
\mathcal{C}_{l m}=[\stackrel{12}{[+}]_{l}+[\stackrel{12}{[-}]_{l}\left[\tau_{m}^{-}+\tau_{m}^{+}\right]
$$

Statement: The C-NOT gate $\mathcal{C}_{l m}$ if applying on $\left|\cdots 0_{l} \cdots 0_{m} \cdots\right\rangle$ transforms it back to the same state, if applying on $\left|\cdots 0_{l} \cdots 1_{m} \cdots\right\rangle$ transforms it back to the same state. If $\mathcal{C}_{l m}$ applies on $\left|\cdots 1_{l} \cdots 0_{m} \cdots\right\rangle$ transforms it to $\left|\cdots 1_{l} \cdots 1_{m} \cdots\right\rangle$, while it transforms the state $\left|\cdots 1_{l} \cdots 1_{m} \cdots\right\rangle$ to the state $\left|\cdots 1_{l} \cdots 0_{m} \cdots\right\rangle$.

Proof: To prove this statement one only has to apply the operator $\mathcal{C}_{l m}$ on states $\left|\cdots i_{l} \cdots i_{m} \cdots\right\rangle,\left|\cdots i_{l} \cdots i_{m} \cdots\right\rangle_{L},\left|\cdots i_{l} \cdots i_{m} \cdots\right\rangle_{R}$, with $i_{l}, i_{m}$ equal 0 or 1 , taking into account equations from Sect. III.

Statement: When applying $\prod_{i}^{n} \mathcal{H}_{i}$ on the $n$ qubit state with all the qubits in the state $\left|0_{i}\right\rangle$, we get the state $\left|\psi_{0}^{0}\right\rangle$

$$
\left|\psi_{0}^{0}\right\rangle=\prod_{i}^{n} \mathcal{H}_{i}\left|0_{i}\right\rangle=\frac{1}{2^{n / 2}} \prod_{i}^{n}\left(\left|0_{i}\right\rangle+\left|1_{i}\right\rangle\right) .
$$

Proof: It is straightforward to prove this statement, if the statement ii. of this section is taken into account. 


\section{USEFUL PROPERTIES OF QUANTUM GATES IN THE TECHNIQUE US- ING NILPOTENTS AND PROJECTORS}

We present in this section some useful relations.

i. One easily finds, taking into account Eqs.(15],17]19]911), the relation

$$
\mathcal{R}_{\Phi_{l}} \mathcal{H}_{l} \mathcal{R}_{\vartheta_{l}} \mathcal{H}_{l}=\frac{1}{2}\left\{\left([+]_{l}+e^{i \varphi_{l}}[-]_{l}\right)\left(1+e^{i \vartheta_{l}}\right)+\left(\tau_{l}^{+}+e^{i \varphi_{l}} \tau_{l}^{-}\right)\left(1-e^{i \vartheta_{l}}\right)\right\},
$$

which transforms $\left|i_{l}\right\rangle$ into a general superposition of $\left|0_{l}\right\rangle$ and $\left|1_{l}\right\rangle$

$$
\begin{aligned}
e^{-i \vartheta_{l}} \mathcal{R}_{\left(\varphi_{l}+\pi / 2\right)} \mathcal{H}_{l} \mathcal{R}_{2 \vartheta_{l}} \mathcal{H}_{l}\left|0_{l}\right\rangle & =\cos \left(\vartheta_{l}\right)\left|0_{l}\right\rangle+e^{i \varphi_{l}} \sin \left(\vartheta_{l}\right)\left|1_{l}\right\rangle, \\
e^{-i\left(\vartheta_{l}-\pi / 2\right)} \mathcal{R}_{\left(\varphi_{l}-\pi / 2\right)} \mathcal{H}_{l} \mathcal{R}_{2 \vartheta_{l}} \mathcal{H}_{l}\left|1_{l}\right\rangle & =\sin \left(\vartheta_{l}\right)\left|0_{l}\right\rangle+e^{i \varphi_{l}} \cos \left(\vartheta_{l}\right)\left|1_{l}\right\rangle
\end{aligned}
$$

ii. Let $\left|\psi_{m}\right\rangle_{p}$ be a general superposition of $p$-qubit states $|k\rangle_{p}=\prod_{l=1, p}\left(\tau_{l}^{-}\right)^{i_{l}}|0\rangle_{l}$, with $i_{l}=0,1$ for a particular choice of $i_{l}$ so that $|k\rangle_{p}$ represents any of the $2^{p}$ basic $p$-qubit states: $\left|\psi_{m}\right\rangle_{p}=\sum_{k=1}^{2^{p}} \alpha_{k}^{m}|k\rangle_{p}$. Then for $\sum_{k=1}^{2^{p}} \alpha_{k}^{m \star} \alpha_{k}^{m}=1$ we find that the operator $\hat{O}_{p}^{m}$ is an involution

$$
\begin{aligned}
\hat{O}_{p}^{m} & =2\left|\psi^{m}\right\rangle_{p}{ }_{p}\left\langle\psi^{m}\right|-I, \\
\left(\hat{O}_{p}^{m}\right)^{\dagger} & =\hat{O}_{p}^{m}, \quad\left(\hat{O}_{p}^{m}\right)^{2}=I .
\end{aligned}
$$

We also find that any operator $\hat{P}_{p}^{k}=I-2 \hat{R}_{p}^{k}$ with $\hat{R}_{p}^{k}=\prod_{l=1}^{p}\left|i_{l}\right\rangle\left\langle i_{l}|=| k\right\rangle_{p}\langle k|$, with a particular choice of $i_{l}$, is also an involution

$$
\begin{aligned}
\hat{P}_{p}^{k} & =I-2 \prod_{l=1}^{p}\left|i_{l}\right\rangle\left\langle i_{l}\right|=I-2 \hat{R}_{p}^{k}, \\
\left(\hat{P}_{p}^{k}\right)^{2} & =\left(I-2 \hat{R}_{p}^{k}\right)^{2}=I .
\end{aligned}
$$

\section{ALGORITHM FOR EXTRACTING PARTICULAR STATES}

Let as define a quantum algorithm, which extracts a particular information out of a data base with $n$ qubits. We assume that the starting state is any superposition of $2^{p}$ states, out of which we are extracting a particular state. In the case that the starting state is a superposition of $2^{p}$ states with equal coefficients (Eq.20), all of them equal to $2^{-p / 2}$, the algorithm is known as the Grover's algorithm [3]. Let $|k\rangle_{p}$ be a state of $p$ qubits

$$
{ }_{p}\langle k \mid m\rangle_{p}=\delta^{k m},
$$


and let $\left|k_{0}\right\rangle_{p}$ be a particular state of p qubits, which we would like to extract out of an general superposition $\left|\psi^{m}\right\rangle$ of $2^{p}$ orthogonal states $|k\rangle_{p}$

$$
\begin{aligned}
\left|\psi^{m}\right\rangle & =\sum_{k=1}^{2^{p}} \frac{\alpha_{k}^{m}}{\sum_{k^{\prime}} \alpha_{k^{\prime}}^{*} \alpha_{k^{\prime}}}|k\rangle_{p}, \\
& =A^{m}\left|k_{0}^{m} \perp\right\rangle_{p}+B^{m}\left|k_{0}\right\rangle_{p},
\end{aligned}
$$

with

$$
\begin{aligned}
{ }_{p}\left\langle k_{0} \mid k_{0}^{m} \perp\right\rangle_{p} & =0, \quad I=\sum_{k}\left|\psi^{k}\right\rangle_{p p}\left\langle\psi^{k}\right| \\
\left|k_{0}^{m} \perp\right\rangle_{p} & =\sum_{k \neq k_{0}} \frac{\alpha_{k}^{m}}{\sqrt{\sum_{k^{\prime} \neq k_{0}} \alpha_{k^{\prime}}^{m \star} \alpha_{k^{\prime}}^{m}}}|k\rangle_{p} \\
A^{m} & =\cos \vartheta_{m}=\frac{\sqrt{\sum_{k^{\prime} \neq k_{0}} \alpha_{k^{\prime}}^{m \star} \alpha_{k^{\prime}}^{m}}}{\sqrt{\sum_{k^{\prime}} \alpha_{k^{\prime}}^{m \star} \alpha_{k^{\prime}}^{m}}}=\sqrt{1-\frac{\alpha_{k 0}^{m \star} \alpha_{k 0}^{m}}{\sum_{k^{\prime}} \alpha_{k^{\prime}}^{m \star} \alpha_{k^{\prime}}^{m}}} \\
B^{m} & =\sin \vartheta_{m} e^{i \varphi_{m}}=\frac{\alpha_{k_{0}}^{m}}{\sqrt{\sum_{k^{\prime}} \alpha_{k^{\prime}}^{m \star} \alpha_{k^{\prime}}^{m}}} .
\end{aligned}
$$

Let us define the operator $\hat{\mathcal{E}}_{p}^{m}$

$$
\hat{\mathcal{E}}_{p}^{m}=\left(2\left|\psi^{m}\right\rangle_{p p}\left\langle\psi^{m}\right|-I\right)\left(I-2\left|k_{0}\right\rangle_{p p}\left\langle k_{0}\right|\right)
$$

We find $\hat{\mathcal{E}}_{p}^{m \dagger} \hat{\mathcal{E}}_{p}^{m}=I$. Since we can write

$$
\begin{aligned}
\left|\psi^{m}\right\rangle_{p p}\left\langle\psi^{m}\right| & =\cos ^{2} \vartheta_{m}\left|k_{0}^{m} \perp\right\rangle_{p p}\left\langle k_{0}^{m} \perp\left|+\sin ^{2} \vartheta_{m}\right| k_{0}\right\rangle_{p p}\left\langle k_{0}\right| \\
& +\sin \vartheta_{m} \cos \vartheta_{m}\left(e^{-i \varphi_{m}}\left|k_{0}^{m} \perp\right\rangle_{p}{ }_{p}\left\langle k_{0}\left|+e^{i \varphi_{m}}\right| k_{0}\right\rangle_{p p}\left\langle k_{0}^{m} \perp\right|\right),
\end{aligned}
$$

it accordingly follows

$$
\begin{aligned}
\hat{\mathcal{E}}_{p}^{m}\left|\psi^{m}\right\rangle_{p} & =\left[\cos 2 \vartheta_{m}+\sin 2 \vartheta_{m}\left(e^{i \varphi_{m}}\left|k_{0}\right\rangle_{p}\left\langle k_{0}^{m} \perp\left|-e^{-i \varphi_{m}}\right| k_{0}^{m} \perp\right\rangle_{p}\left\langle k_{0}\right|\right)\right]\left|\psi^{m}\right\rangle_{p} \\
& =\cos \left(3 \vartheta_{m}\right)\left|k_{0}^{m} \perp\right\rangle_{p}+e^{i \varphi_{m}} \sin \left(3 \vartheta_{m}\right)\left|k_{0}\right\rangle_{p} .
\end{aligned}
$$

If we apply the operator $\hat{\mathcal{E}}_{p}^{m} j$ times, we find

$$
\begin{aligned}
\left(\hat{\mathcal{E}}_{p}^{m}\right)^{j}\left|\psi^{m}\right\rangle_{p} & =\left[\cos \left(2 j \vartheta_{m}\right)+\sin \left(2 j \vartheta_{m}\right)\left(e^{i \varphi_{m}}\left|k_{0}\right\rangle_{p}\left\langle k_{0}^{m} \perp\left|-e^{-i \varphi_{m}}\right| k_{0}^{m} \perp\right\rangle_{p}\left\langle k_{0}\right|\right)\right]\left|\psi^{m}\right\rangle_{p} \\
& =\cos \left[(2 j+1) \vartheta_{m}\right]\left|k_{0}^{m} \perp\right\rangle_{p}+e^{i \varphi_{m}} \sin \left[(2 j+1) \vartheta_{m}\right]\left|k_{0}\right\rangle_{p}
\end{aligned}
$$

It follows that $\left(\hat{\mathcal{E}}_{p}^{m}\right)^{j}$ extracts our particular state $\left|k_{0}\right\rangle_{p}$ out of the initial state $\left|\psi^{m}\right\rangle_{p}$, if we choose $j$ so that

$$
\sin \left[(1+2 j) \vartheta_{m}+\varepsilon\right]=\frac{\pi}{2}
$$


for as small $|\varepsilon|$ as possible. If we choose the initial state $\left|\psi^{m}\right\rangle_{p}$ to be just our desired state, then $\vartheta_{m}=\pi / 2$ and $j=0$. If the initial state has all the coefficients equal to $2^{-p / 2}$, then this is the Grover's algorithm [3], provided that $\sin \vartheta_{m}=2^{-p / 2}$.

\section{CONCLUDING REMARKS}

We presented in this paper how can the Clifford algebra technique [1, 2] be used in quantum computers for generating quantum gates, demonstrating that the Clifford algebra technique 1 1, 2] makes the formation of quantum gates very transparent. Although our projectors and nilpotents can as well be expressed in terms of the ordinary projectors and the ordinary operators (we chose the phases so that when going into the matrix representation the usually used matrices are reproduced), the elegance of the technique is helpful to better understand the operators appearing in the quantum gates and quantum algorithms.

We present also the algorithm appropriate for extracting an information out of any superposition of $2^{n} n$ qubit states. This is a generalization of the well known Grover's algorithm, allowing in principle a faster extraction of a particular information than the Grover's algorithm, if the initial state favours the particular state. Our algorithm becomes the Grover's algorithm for a very special choice of the initial state, out of which the information is extracting.

The presented Clifford algebra technique (with nilpotents and projectors[1, 2]) used in this paper for quantum gates and algorithms for the two states quantum bits can be quite easily generalized to cases with more than two states qubits.

[1] N. S. Mankoč Borštnik, H. B. Nielsen, J. of Math. Phys. 43, 5782 (2002), hep-th/0111257.

[2] N. S. Mankoč Borštnik, H. B. Nielsen, J. of Math. Phys. 44, 4817 (2003), hep-th/0303224.

[3] L. K. Grover, Proceedings, 28th Annual ACM Symposium on the Theory of Computing, 212 (1996), quant-ph/9605043.

[4] S. S. Somaroo, D. G. Cory, T. F. Havel, Phys. Lett. A 240,1 (1998), quant-ph/9801002.

[5] T. H. Havel, C. J. L. Doran, AMS Contemporary Math, Quantum Computation and Quantum Information Science (S. Lomonaco, ed.). (2000) quant-ph/0004031. 